\title{
AVANZA LA POLÍTICA PÚBLICA DE MEDIOS \\ ALTERNATIVOS,INDEPENDIENTES, COMUNITARIOS Y CIUDADANOS DE MEDELLÍN
}

\section{Mónica Valle F.}

Destino en este espacio algunos párrafos para exponer lo que considero avances en la formulación de la Política Pública de Medios Alternativos, Independientes, Comunitarios y Ciudadanos de Medellín, proceso que he visto gestarse y que está ad portas de su implementación. Pero antes de ello les presento brevemente los contenidos de esta edición de la Revista Luciérnaga - Comunicación.

En esta publicación diversos autores proponen que el cine, la ficción televisiva y el talk show pueden utilizarse como estrategias formativas, recursos para analizar acontecimientos del pasado y como argumentos para pensar la sociedad; se reflexiona en torno al ocaso de los héroes en las narrativas contemporáneas. Análisis acerca del periodismo deportivo y el ciberperiodismo, también hacen parte de esta edición N16. Los artículos provienen de investigadores de Ecuador, Uruguay, México y Colombia. Les invitamos a leer, debatir, compartir estos abordajes y resultados investigativos así como a presentar sus escritos para los números 17 y 18.
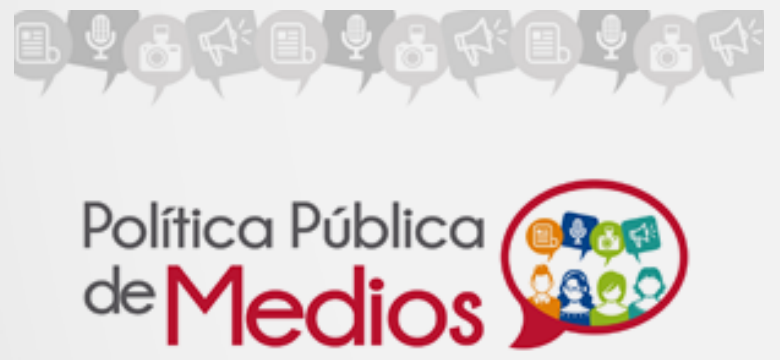

Alternativos, Independientes, Comunitarios y Ciudadanos de Medellin

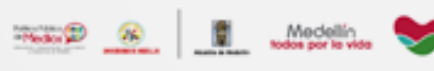

En Latinoamérica la referencia a la comunicación alternativa, popular, ciudadana, comunitaria, ha estado vinculada a los movimientos sociales, a la crítica de la sociedad imperante, la educación y también al desarrollo sociocultural de las comunidades. Entre los maestros de maestros que han generado conocimiento en torno a este tipo de comunicación están Luis Ramiro Beltrán (Bolivia), Rosa María Alfaro (Perú), Alfonso Gumucio Dagron (Bolivia), Amparo Cadavid Bringe (Colombia), Mario Kaplún (Argentina) y su hijo Gabriel Kaplún (Uruguay) entre muchos otros que no solo la investigan sino que la viven e implementan en sus comunidades.
Aunque Colombia podría considerarse uno de los países más avanzado en términos de la legislación de medios, los derechos de los denominados medios comunitarios han debido conseguirse con firmeza, constancia y paso a paso. La Carta Constitucional de Colombia de 1991, en el Artículo 20, contempla que:

\begin{abstract}
"Se garantiza a toda persona la libertad de expresar y difundir su pensamiento y opiniones, la de informar y recibir información veraz e imparcial, y la de fundar medios masivos de comunicación. Estos son libres y tienen responsabilidad social. Se garantiza el derecho a la rectificación en condiciones de equidad. No habrá censura".
\end{abstract}

En el 2002, se expidió la Resolución $N^{\circ} 719$, siendo Ministro de Cultura el antioqueño Juan Luis Mejía Arango, actual rector de la Universidad EAFIT, reconocido por su vinculación a la cultura y a la educación. Por medio de esta, se declara a los medios de comunicación ciudadanos y/o comunitarios como una expresión cultural de la nacionalidad colombiana. En tanto se considera que:

[son] espacios de creación cultural desde lo local y regional que contribuyen a la manifestación de imágenes plurales de nación; espacios de expresión y difusión cultural que inciden implícita y explícitamente en la construcción de la realidad colombiana y sus referentes...su carácter sobrepone los intereses colectivos sobre los individuales... son espacios de lo público que inciden en las practicas ciudadanas; están generando productos y procesos culturales que redundan en el fortalecimiento de la identidad cultural de la región y en la construcción del proyecto de nación...se han desarrollado en los últimos años cuantitativa y cualitativamente, y han alcanzado una cobertura nacional con la participación de las diferentes comunidades del territorio [1] 


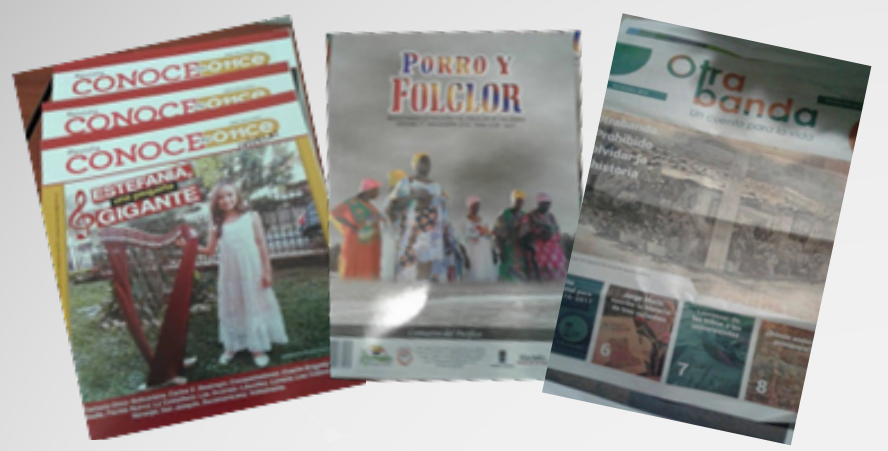

La idea de una política pública para medios alternativos de la ciudad de Medellín se presenta como una de las recomendaciones del Proyecto Estructuración de la Red de Medios Alternativos para la Convivencia Ciudadana (2006), en el que participé como investigadora [2], [3]. Este estudio lo encargó la Alcadía de la ciudad, con recursos del BID, a la Caja de Compensación Comfama [4], era el segundo desarrollado en la región; el primero "Comunicación, Vida y Territorio" CONVITE, lo realizó la Gobernación de Antioquia, en el año 2005. Este proyecto fue liderado por el Instituto para el Desarrollo de Antioquia (IDEA), con el apoyo de la $U$ de $A$ y la caja de compensación Comfenalco con el objetivo de fortalecer las capacidades conceptuales, administrativas, técnicas y de cooperación de los medios [5]. Ese mismo año, el interés por los medios comunitarios llegó a Bogotá, y en noviembre la Alcadía Mayor realizó el "Encuentro Distrital de Comunicación Comunitaria [6], cuyo resultado fue la conformación de la Mesa Bogotana de Comunicación desde donde se elaboró el "Documento de Propuestas de Lineamientos de Políticas en Comunicación Comunitaria para Bogotá" en el que se planteó, entre otros aspectos:

"Inclusión de los medios locales, comunitarios, centros de producción radial comunitarios y colectivos de la comunicación, en la contratación para la divulgación de las campañas comunicativas de promoción de proyectos y programas institucionales del distrito, teniendo en cuenta el potencial de acción de estos medios en términos de su acceso a audiencias locales específicas" [7].

En el 2011, conjuntamente con estudiantes del Politécnico Colombiano Jaime Isaza Cadavid, organizamos la primera exhibición de medios alternativos, evento que contó, con dos versiones más en el año 2012.
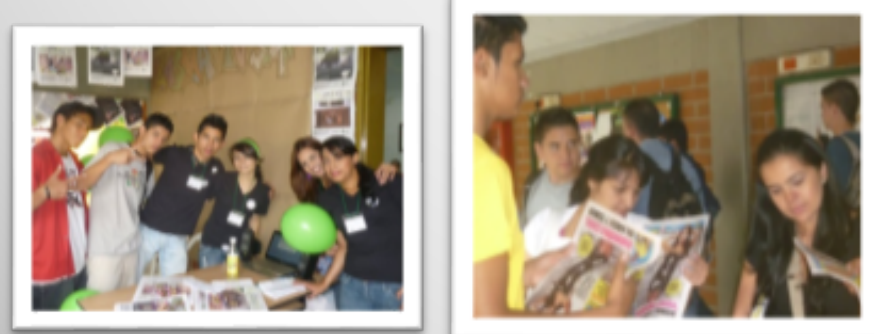

En el 2013, El Consejo de Medellín expide el Acuerdo 73 [8] mediante el cual dicta los lineamientos para la creación de la Política Pública de estos medios:

"dirigida a promover y fortalecer los procesos comunitarios de comunicación alternativos, independientes, comunitarios y ciudadanos como expresión de la participación, la cultura ciudadana y la divulgación democrática de opiniones" (Art. 1).

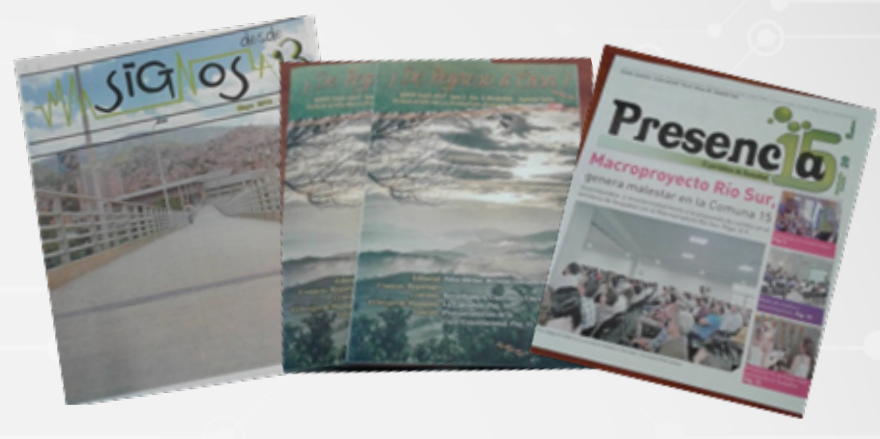

Las líneas estratégicas que guían la política son la creación y fortalecimiento de las redes de medios, la capacitación, la visibilización y el acceso a estos. Los principios que le inspiran: el fomento al derecho de la información y a la libre expresión, la participación ciudadana, identificación, inclusión, articulación, autonomía, coordinación, corresponsabilidad, seguimiento y evaluación (Art. 3. Acuerdo 73 de 2013).

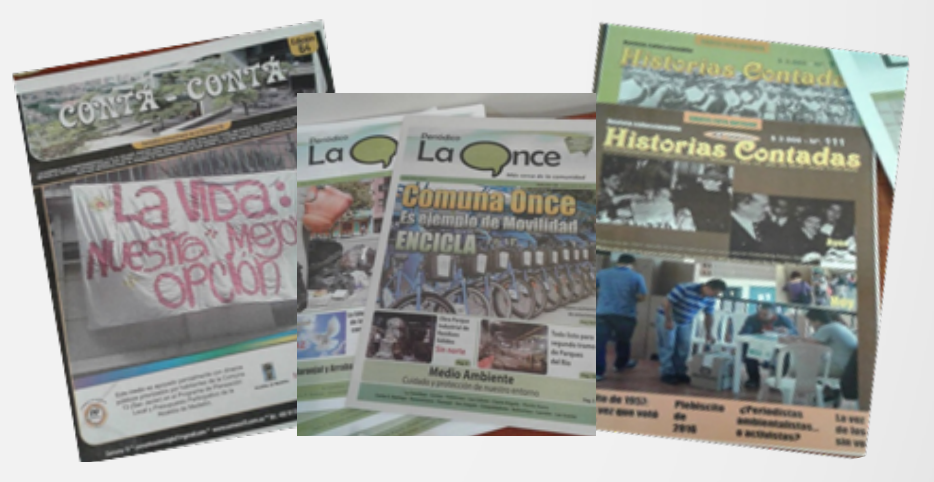

En el 2015, la Alcaldía, encomienda a la Universidad de Medellín la realización de otro Diagnóstico de medios alternativos [9]. Durante el gobierno de ese año, se adelantaron algunos procesos en torno a la formulación de la Política, pero es en el Plan de Desarrollo Municipal "Medellín Cuenta con vos" (2016-2019) que se asume el reto "Medellín Participativa" y se crea el proyecto denominado "Medios ciudadanos para la interacción y movilización social" con el fin de formular e implementar la política para estos [10], resaltando que se pretende también proteger la identidad y la memoria de dichos medios. 

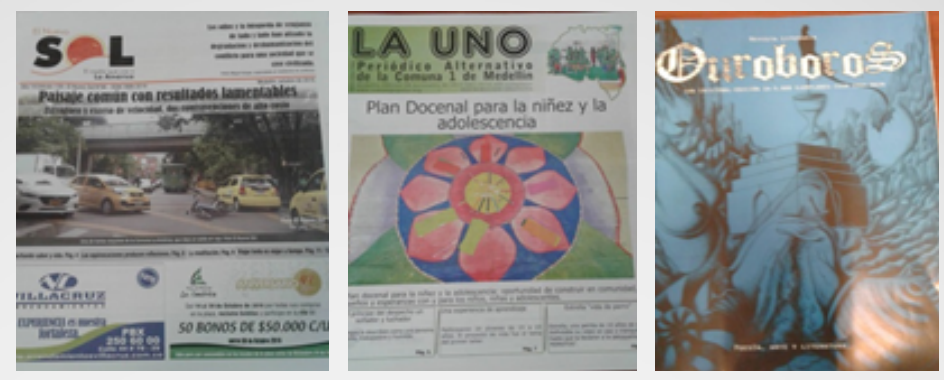

A partir de ese momento se retoma la mesa articuladora, la cual queda integrada por medios, gremios y asociaciones del sector de la comunicación, participan también EPM, el Politécnico Colombiano Jaime Isaza Cadavid y las Universidades de Antioquia, UNAD, Luis Amigó, Uniminuto y la Pontificia Bolivariana.

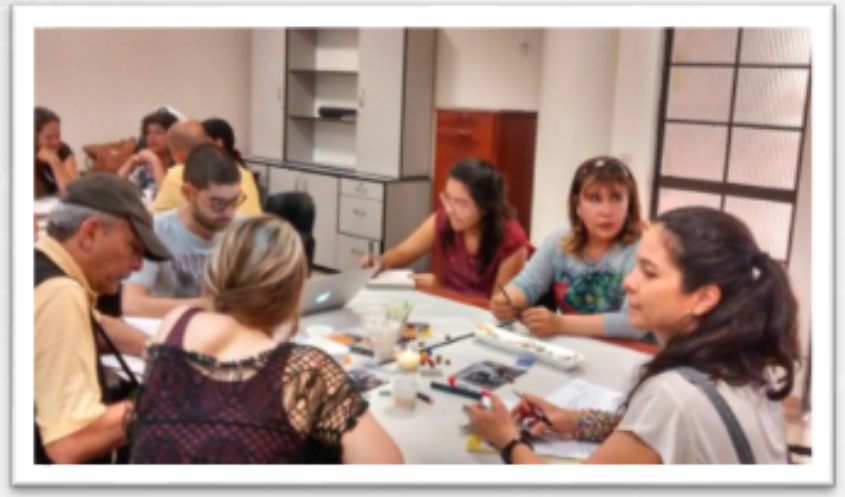

Los Medios aportan a la ciudad: transformación, conocimiento,movilización, pluralismo, integración, transparencia, identidad, consolidación de tejido social.

En la formulación de la política se ha seguido la ruta propuesta por Diana Álvarez Restrepo de la Secretaria de comunicaciones, la cual se esboza en el siguiente gráfico.
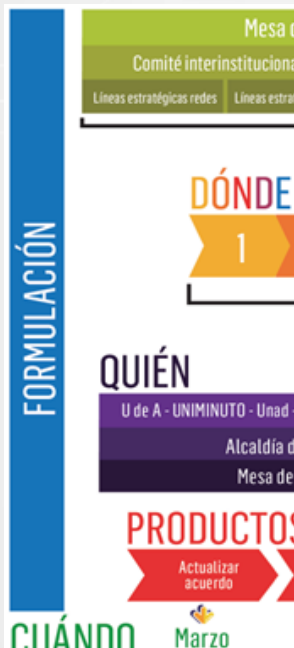

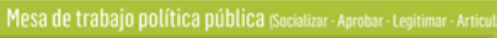
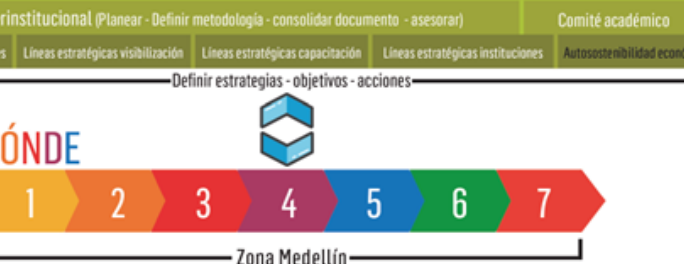

CUÁNDO Marzo
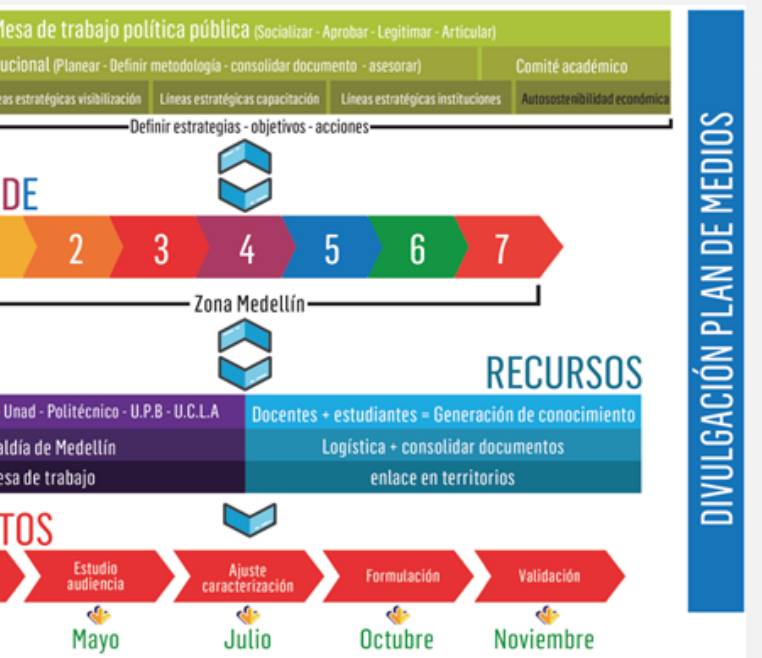

Tomado de informe Mesa Articuladora 2016

La construcción de esta Política ha implicado diversos foros, "se entiende que éstos son el escenario para que el debate y el consenso permitan que las líneas estratégicas del Acuerdo 73, puedan ser objeto de definiciones, profundizaciones y una prospectiva que consulte con el espíritu que anima el proceso" [11]. La estructuración de estos puede consultarse en el link que aparece a continuación: http://www.politicamediosmed.com/foros-de-discusion

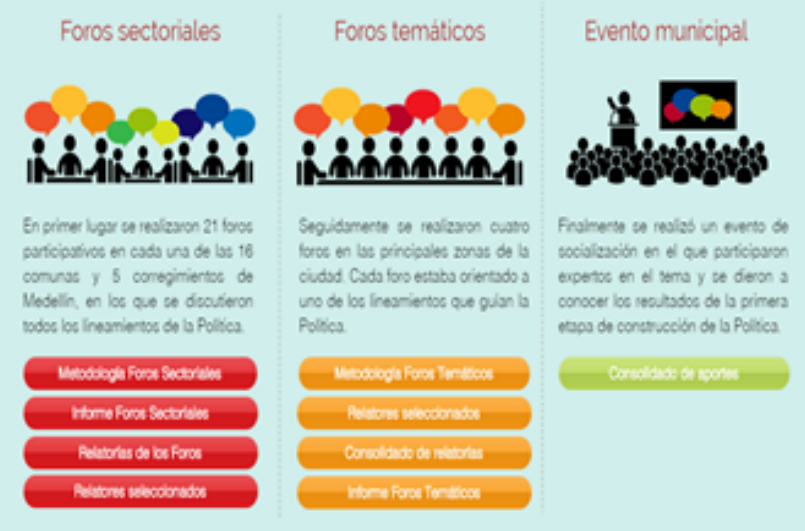

El Sexto Encuentro de Periodismo Comunitario, que tuvo lugar entre el 16 y 17 de noviembre de 2016, también se enmarcó en el proceso de construcción de esta política. Se realizaron recorridos por la ciudad para conocer la experiencia de los medios ubicados en las diferentes comunas de Medellín. "Formar, informar, inconformar y transformar" es la misión de los medios comunitarios expresan 
los líderes del Periódico "Mi Comuna 2". En el Encuentro Gabriel Kaplún expuso acerca de la Comunidad, comunicación y la sustentabilidad de dichos medios.

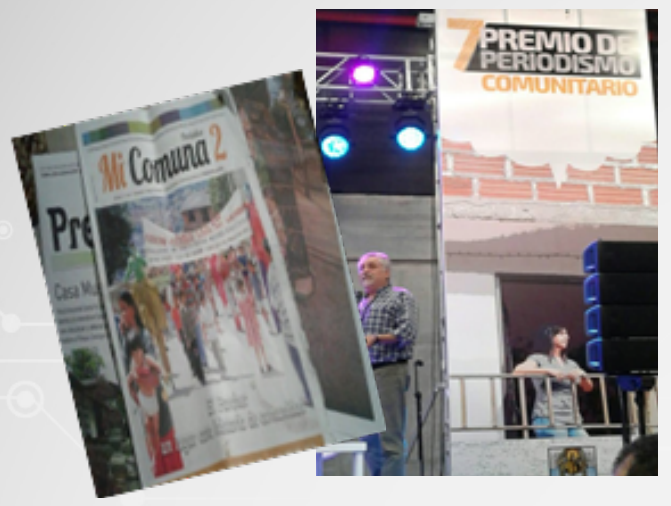

Para una mayor visibilización de estos medios, TeleMedellín a través del programa "Mi Barrio Cuenta" [12] les ha vinculado en la producción y realización del seriado. Este Canal Municipal también está capacitando a los medios en producción, edición y realización audiovisual. El Politécnico Colombiano Jaime Isaza Cadavid está realizando el registro audiovisual del proceso llevado a cabo en la formulación de la política y produce el Webdocumental de los medios direccionados desde la Corporación el Megáfono ubicada en la comuna 1 de Medellín; el realizador es el comunicador audiovisual Jose González Arteaga.

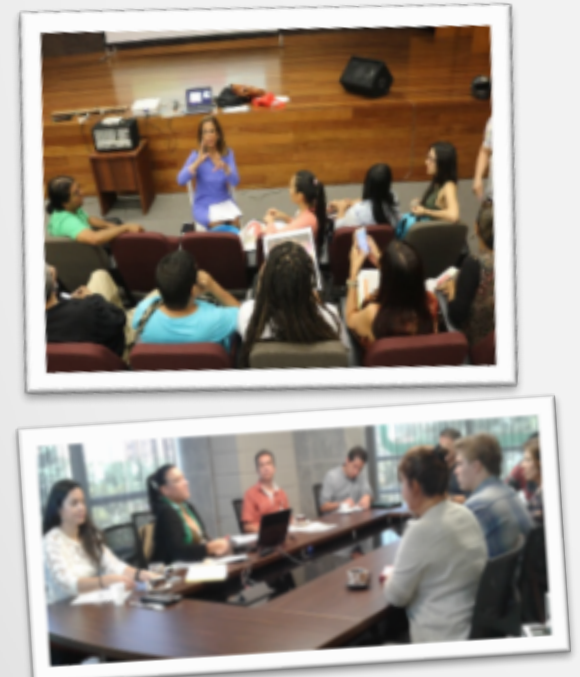

La construcción de la Política Pública de estos medios también ha pasado por el debate del género. En foro realizado, se abordaron temáticas acerca de la mujer como sujeto de noticia, la importancia de la perspectiva de género y la necesidad de equipos de trabajos mixtos en los medios comunitarios.
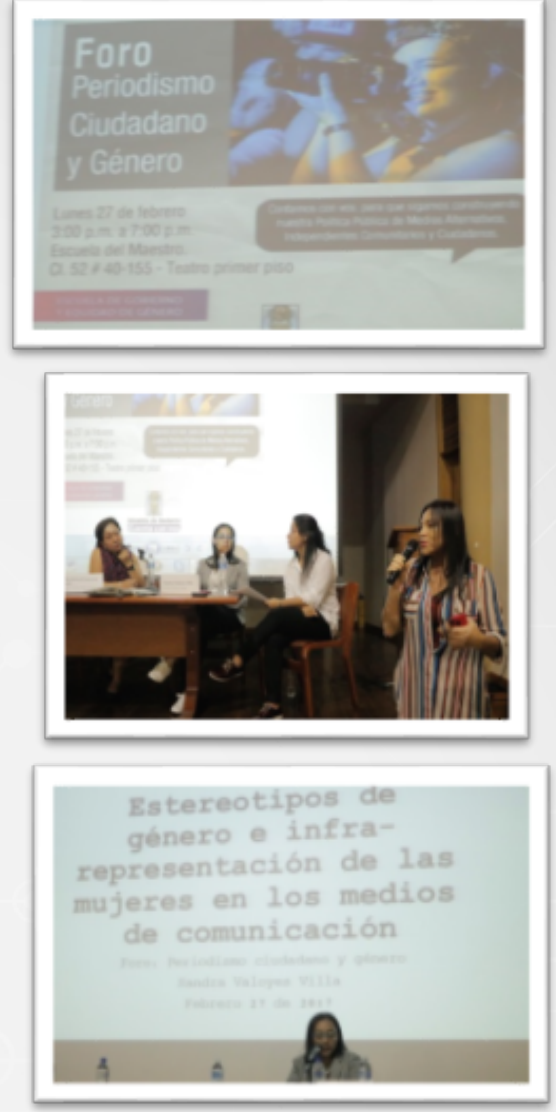

La socialización de este proceso se realiza con el acompañamiento de los integrantes de las mesas de trabajo. La participación por comuna a los foros se puede observar en el siguiente mapa.

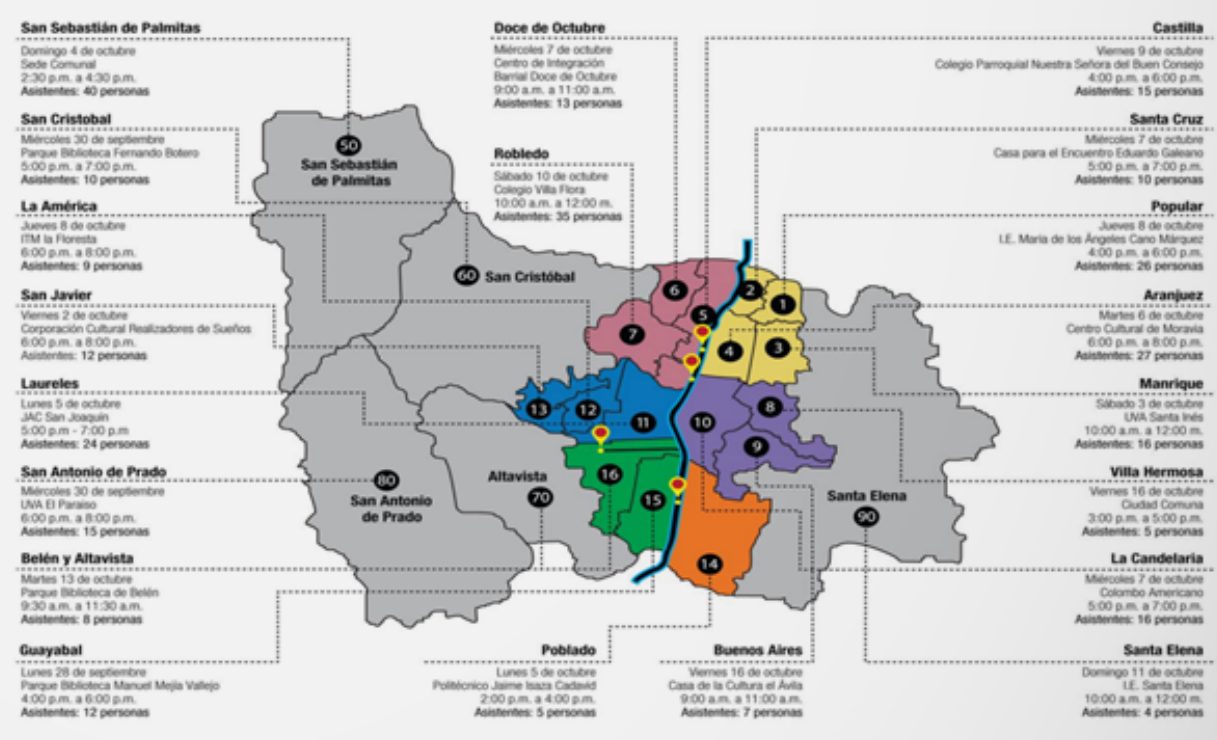

Tomado de: http://www.politicamediosmed.com/foros-de-discusion 
Para la identificación y reconocimiento de las dinámicas adelantadas en el proceso de construcción de esta política, la Secretaria de comunicaciones ha compartido algunas definiciones para los participantes de las Mesas de trabajo. Se entiende la comunicación popular como la vinculada con la acción de los movimientos y organizaciones populares que implementan y desarrollan sus propios canales. Dependiendo del tipo de práctica social que la orienta. A esta también se le denomina alternativa, participativa, horizontal, comunitaria y dialógica.

Se ha definido la comunicación pública como aquella que genera procesos de movilización social para el reconocimiento de problemas y la superación de estos mediante una agenda común. Se pretende con este tipo de comunicación construir contenidos, imaginarios, propósitos realizables y pertinentes para los diversos actores sociales, conservando el respecto a su autonomía e independencia cultural, ideológica o política.

Una vez formulada y socializada la Política Pública de Medios Alternativos, Independientes, Comunitarios y Ciudadanos de Medellín, se espera su implementación para mediados del 2018.

\section{Mónica Valle F.}

\section{Editora}

Integrante Mesa Articuladora Política Pública de Medios

\section{Notas}

[1] Resolución N 719 de 2000. Medios Comunitarios como expresión de la nacionalidad. Material Bibliográfico. Política Pública de Medios. http:// www.politicamediosmed.com/bibliografia (Hernán Atehortúa). Disponible en http://media.wix.com/ ugd/a69_301b6952dfb94950b536a4532d38a155. pdf

\section{[2] Valle, Mónica María (2012). MEDIOS} ALTERNATIVOS DE MEDELLIN [1]: Recuento Histórico (1987-2006). Revista Virtual Luciérnaga, Año 4, N7. Grupo de Investigación en Comunicación, Facultad de Comunicación Audiovisual, Politécnico Colombiano Jaime Isaza Cadavid. MedellínColombia. ISSN 2017-1557. Págs. 36-46. http://www.politecnicojic.edu.co/images/downloads/ publicaciones/revista-luciernaga/luciernaga-07/ pdf/4_medios_alternativos.pdf
[3] Valle, Mónica María (2012). MEDIOS ALTERNATIVOS DE MEDELLIN [2]: Diagnóstico 2005-2006. Revista Virtual Luciérnaga, Año 4, N8. Grupo de Investigación en Comunicación, Facultad de Comunicación Audiovisual, Politécnico Colombiano Jaime Isaza Cadavid. MedellínColombia. ISSN 2027-1557. Págs. 57. Disponible en. http://www.politecnicojic.edu.co/images/downloads/ publicaciones/revista-luciernaga/luciernaga-08/pdf/ medios-alternativos.pdf

[4] COMFAMA (2007). https://es.scribd.com/ doc/6919283/MEDIOS-DE-COMUNICACIONALTERNATIVOS-PARA-LA-CONVIVENCIA

[5] Proyecto "Comunicación, Vida y Territorio" CONVITE (2005). http://documentslide.com/ documents/comunicacion-vida-y-territorio-proyectopara-el-fortalecimiento-de-los-medios-localesde-comunicacion-por-la-equidad-y-la-vida-enantioquia-taller-de-produccion.html

[6] Encuentro Distrital de Comunicación Comunitaria (2005). Alcaldía Mayor de Bogotá D.C. 12 y 13 de diciembre. Auditorio Alfonso López PumarejoUniversidad Nacional. Bogotá- Colombia.

[7] "Documento de Propuestas de Lineamientos de Políticas en Comunicación Comunitaria para Bogotá" (2007). Disponible en http://media.wix.com/ ugd/a69_25f19e4e7b0045488597f57456586c0c.pdf

[8] Gaceta Oficial N4207. Pág. 28 https:// www.medellin.gov.co/irj/go/km/docs/pccdesign/ SubportaldelCiudadano_2/PlandeDesarrollo_0_15/ Publicaciones/Shared $\% 20$ Content/GACETA $\% 20$ OFICIAL/2014/Gaceta\%204207/4207.pdf

[9] Diagnóstico de medios alternativos, Independientes, Comunitarios y Ciudadanos de Medellín, U de M (2015). Disponible en http://media.wix.com/ugd/ a69_03edf131ca3846adad1445edf4b3d2d6.pdf

[10] Plan de Desarrollo de Medellín "Medellín cuenta con vos" ( 2016 -2019).

http://www.medellincomovamos.org/download/ presentacion-plan-de-desarrollo-de-medellin-20162019-medellin-cuenta-con-vos-2016

[11] Diana Álvarez Restrepo de la Secretaria de comunicaciones de la Alcaldía de Medellín

[12] Mi Barrio Cuenta - Capítulo 8 emitido el 28 de diciembre de 2016. https://www.youtube. com/watch?v=ooThX7kJV3U 


\section{Para citar este texto:}

Valle, F. Mónica (2016). Avanza la Política Pública de Medios Alternativos, Independientes, Comunitarios y Ciudadanos de Medellín. Revista Luciérnaga/ comunicación Año 8, N16. Facultad de Comunicación Audiovisual- Politécnico Colombiano Jaime Isaza Cadavid- PCJIC \& Facultad de Ciencias de la Comunicación - Universidad Autónoma de San Luis Potosí- UASLP. México. Págs. 4-9. DOI. 10.33571/revistaluciernaga.v8n16a1 